\title{
A computation of modular forms of weight one and small level
}

\author{
Kevin Buzzard ${ }^{1}$ • Alan Lauder ${ }^{2}$
}

Received: 30 August 2016 / Accepted: 2 October 2016/ Published online: 10 November 2016

(C) The Author(s) 2016. This article is published with open access at Springerlink.com

\begin{abstract}
We report on a computation of holomorphic cuspidal modular forms of weight one and small level (currently level at most 1500) and classification of them according to the projective image of their attached Artin representations. The data we have gathered, such as Fourier expansions and projective images of Hecke newforms and dimensions of space of forms, is available in both Magma and Sage readable formats on a webpage created in support of this project.
\end{abstract}

Résumé Nous faisons état de calculs de formes modulaires paraboliques (aussi dites cuspidales) holomorphes de poids 1 et de petits niveaux (au plus 1500 à ce stade-ci) et nous les classifions selon les images projectives des représentations d'Artin attachées à ces formes. On trouvera sur la page Web les informations obtenues, comme les développements en séries de Fourier et les dimensions de ces espaces de formes, sous forme de tableaux faciles à lire et créés dans le cadre de ce projet via Magma et Sage.

Keywords Modular form · Weight one - Galois representation

Mathematics Subject Classification $11 \mathrm{~F} 11 \cdot 11 \mathrm{~F} 80$

Alan Lauder

lauder@maths.ox.ac.uk

Kevin Buzzard

k.buzzard@imperial.ac.uk

1 Department of Mathematics, Imperial College London, 180 Queen's Gate, London SW7 2AZ, England, UK

2 Mathematical Institute, University of Oxford, Woodstock Road, Oxford OX2 6GG, England, UK 


\section{The computation and some observations}

The theory and practice of computing weight one modular forms has typically lagged behind that of computing higher weight forms. This is mainly because forms of higher weight are cohomological and there is a direct method for computing them using modular symbols. Indeed, the computer algebra package Magma [1] has been able to compute forms of weight two or more for over fifteen years now using modular symbols, and the free open source package Sage [5] can also compute these forms. (Custom code existed well before then: examples we know of are due to Cohen-Skoruppa-Zagier, Cremona, Gouvêa and Stein.) By contrast, no such direct method is known in weight one and there were no generally applicable algorithms until more recently-the pioneering work of Buhler [3] and project coordinated by Frey [9] were both focused on computing one or more specific spaces of forms, rather than on a systematic computation.

The first author adapted the methods of Buhler and Frey et al. so that they could be applied systematically, and reported on the details of the algorithm in [4]. This code, which computed bases of spaces of modular forms of weight one and arbitrary Dirichlet character, was written in Magma and incorporated into the distributed version of the Magma package by Steve Donnelly. The authors have used this code (with some additions) to carry out a computation of the Hecke newforms in weight one for increasing level and all characters. Computations have been completed for all levels up to 1500 and the data obtained is available in both Magma and Sage formats on a webpage which accompanies this paper [2]. George Schaeffer informs the authors that he has implemented his more efficient "Hecke stability method" [12] for weight one in Sage, but only for quadratic character, and has computed such newforms up to level around 800. A Magma implementation of Schaeffer's algorithm for general character would be of great practical use in extending our tables of weight one modular forms (at present Sage seems a less suitable platform for carrying out such computations).

What do we mean by computing weight one newforms? For a given level $N$ and odd character $\chi$, we present each cuspidal new eigenform $f \in S_{1}(N, \chi)$ as a truncated $q$ expansion $f(q)+\mathcal{O}\left(q^{M}\right)$ with Fourier coefficients in an explicit abelian field containing the image of $\chi$. The $q$-adic precision $M$ is chosen so that there is a unique weight two form of level $N$ and trivial character whose $q$-expansion is $E_{1}\left(1, \chi^{-1}\right) \cdot f(q)+\mathcal{O}\left(q^{M}\right)$, where $E_{1}\left(1, \chi^{-1}\right)$ denotes the Eisenstein series of weight one and characters 1 and $\chi^{-1}$, thus ensuring further Fourier coefficients can be easily computed if desired using modular symbols in weight two. On our webpage bases of spaces of modular forms are given in the same manner, and we provide code which allows the user to compute the Fourier expansions to arbitrary precision, as well as computations of $q$-expansions up to $O\left(q^{10,000}\right)$.

Having computed all newforms up to a given level, two natural questions for us to consider were what further computations can one do with this data, and how to make the data available to other researchers in an easily accessible manner.

The main computation we did with the data was that for each cuspidal newform we rigorously computed whether the projective image of the associated Galois representation was a dihedral group or one of $A_{4}, S_{4}$ or $A_{5}$. This seemed like a natural question to ask and it needed, what was for us, a novel trick to answer. Note that as a consequence we are able to determine the smallest level $N$ for which there exists a weight one modular form whose associated projective Galois representation has image $A_{5}$. The level is 633 and the Dirichlet character has order 10. The analogous questions for $A_{4}$ and $S_{4}$ were answered in [4], levels 124 
and 148 with characters of order 6 and 4, respectively. This level 633 icosahedral form does not seem to have been computed before-Buhler's original icosahedral example had level 800 and the first author in [4] found an example with level 675. (The original motivation for the second author in carrying out such computations was for a specific experimental application which required knowledge of the projective image. Such a classification of cuspidal newforms of small level in weight one was crucial in developing and numerical testing the conjectural, and occasionally provable, new constructions of points on elliptic curves and units defined over dihedral, $A_{4}, S_{4}$ and $A_{5}$ number fields in [6,7]. The $A_{4}$ form of level 124, and the $S_{4}$ form of level 148 occur in [6, Examples 5.4 and 5.6].)

One further computation which could be done with the data is to find the number field cut out by the projective Galois representation associated to each cuspidal newform. The most straightforward way to answer this question for a given newform is to search in a precomputed table of number fields. Once a number field has been found that one suspects is the right one, one can rigorously prove that it is by invoking the Artin conjecture, which is known in this situation thanks to the work of Khare and Wintenberger [10]. For example, the number field cut out by the projective Galois representation attached to the $A_{5}$ form in level 633 is the splitting field of the polynomial $x^{5}-211 x^{2}-1266 x-1899$. We did not attempt to automate this process though. (An alternative analytic approach to finding candidate number fields, working directly from the Fourier expansions, is to invoke Stark's conjecture [13]. This may also be used to find the maximal real subfield of the field cut out by the Galois representation itself.)

We have made available on a webpage all of our data (on Fourier expansion of cuspidal newforms, dimensions and bases of spaces of cuspidal forms) in easily readable Magma and Sage format, along with accompanying code which allows the user to perform some further computations [2]. This seemed to the authors the best way of making the data accessible and useable for other researchers (including developers of the L-functions and modular forms database [11]).

In this note we shall not discuss the methods used to compute the newforms in each level: this was an involved computation, and took many months, but the most interesting aspects of the methods used are already described in [4]. The remainder of this note is devoted solely to explaining how the projective image of each cuspidal newform was rigorously determined. We hope though that the reader will view this paper and the accompanying website as two halves of a whole, and explore the code and data on the website alongside reading this short paper.

\section{Determining the projective image}

It is a simple matter to guess the projective image of the Galois representation attached to a weight one modular form, given the initial Fourier coefficients. Moreover, an effective version of the Cebotarov density theorem allows one to turn this guess into a proof, given sufficiently many of these Fourier coefficients. Such an approach is unlikely to lead to a very practical test though: too many Fourier coefficients would be required. Our purpose is to present some theoretical criteria involving Fourier coefficients which allow one to rigorously distinguish between the different projective images, and which are not dependent on an effective Cebotarov density theorem and so require relatively few Fourier coefficients and work well in practice. To explain these criteria we need first set up some notation. 


\subsection{Weight one newforms and Galois representations}

Let $N \geq 1$ and $\chi$ be a Dirichlet character modulo $N$. The space of weight 1 cuspforms of level $\Gamma_{1}(N)$ and character $\chi$ is finite-dimensional over the complex numbers and will be denoted $S_{1}(N, \chi)$. If $f=\sum_{n>1} a_{n} q^{n} \in S_{1}(N, \chi)$ then we say $f$ is a normalised eigenform if $f$ is an eigenform for all the Hecke operators (including those at the bad primes) and $a_{1}=1$. As for higher weights there is a theory of oldforms and newforms. If $f=\sum a_{n} q^{n} \in S_{1}(N, \chi)$ is a normalised eigenform which is furthermore a newform of level $N$, then a theorem of Deligne and Serre (Theorem 4.1 of [8]) tells us that there is a continuous odd irreducible Galois representation

$$
\rho_{f}: \operatorname{Gal}(\overline{\mathbf{Q}} / \mathbf{Q}) \rightarrow \mathrm{GL}_{2}(\mathbf{C})
$$

associated to $f$. In contrast to the higher weight case, the target here is a complex group rather than a $p$-adic one, and furthermore the image of $\rho_{f}$ is finite, so $\rho_{f}$ can be thought of as a faithful representation $\operatorname{Gal}\left(M_{f} / \mathbf{Q}\right) \rightarrow \mathrm{GL}_{2}(\mathbf{C})$, where $M_{f}$ is a finite Galois extension of $\mathbf{Q}$. The representation $\rho_{f}$ has conductor $N$, and in particular the extension $M_{f} / \mathbf{Q}$ is unramified outside $N$. The representation $\rho_{f}$ is characterised by the following property: if $p \nmid N$ is prime, then the characteristic polynomial of $\rho_{f}\left(\right.$ Frob $\left._{p}\right)$ is $X^{2}-a_{p} X+\chi(p)$.

We can projectivise $\rho_{f}$ and obtain a projective Galois representation

$$
\bar{\rho}_{f}: \operatorname{Gal}(\overline{\mathbf{Q}} / \mathbf{Q}) \rightarrow \mathrm{PGL}_{2}(\mathbf{C}) .
$$

The image of $\bar{\rho}_{f}$ is a finite subgroup of $\mathrm{PGL}_{2}(\mathbf{C})$ and is hence either cyclic, dihedral (including the degenerate case of the non-cyclic group of order 4), or isomorphic to $A_{4}, S_{4}$ or $A_{5}$. (This argument goes back to Weber; the pre-image of a finite subgroup of $\mathrm{PGL}_{2}(\mathbf{C})$ in $\mathrm{SL}_{2}(\mathbf{C})$ stabilises a hermitian form so lives in $S U$ (2), which maps in a 2-to-1 manner onto $\mathrm{SO}_{3}(\mathbf{R})$, and the finite subgroups of this can be classified via the Platonic solids.) Because $\rho_{f}$ is irreducible, the image of $\bar{\rho}_{f}$ cannot be cyclic (otherwise the image of $\rho_{f}$ would be a central extension of a cyclic group by a cyclic group and hence abelian), but the other cases do occur. We refer to $f$ in these cases as a dihedral form, an $A_{4}$ form, an $S_{4}$ form or an $A_{5}$ form respectively. If $f$ is not a dihedral form then we say it is exotic.

In our computations, based upon the algorithms in [4], the dihedral forms in each level were separately computed using explicit class field theory as a step towards determining a basis for the whole space of modular forms in that level. Note in particular that magma's inbuilt class field theory algorithms can be used to rigorously compute the space of diheral forms of a given level (and these algorithms run very quickly in practice for levels at most 1500). All the newforms in each level were later computed, including recomputing the dihedral ones, when the newspace in that level was separated into eigenforms using Hecke operators. So for us the main problem left was to quickly and rigorously sort the exotic forms. One could adopt a similar approach here as for the dihedral case, but to do this one would have to solve the following sort of problem: given a finite set of primes $S$, we would need to be able to produce a provably complete list of Galois extensions of $\mathbf{Q}$ which were unramified outside $S$ and had Galois group $A_{5}$ (say). For certain sets of primes $S$ such tables exist (for example the Jones-Roberts database) but we wanted to completely automate the process within magma so we had to come up with another approach.

\subsection{Separating out the exotic forms into $A_{4}, S_{4}$ and $A_{5}$ forms}

We start with a simple lemma. 
Lemma 1 Let $g \in \mathrm{PGL}_{2}(\mathbf{C})$ and $\tilde{g} \in \mathrm{GL}_{2}(\mathbf{C})$ be any lift of $g$.

(a) If $g$ has finite order $n$ then the complex number $c(\tilde{g})=\operatorname{trace}(\tilde{g})^{2} / \operatorname{det}(\tilde{g})$ is independent of the choice of $\tilde{g}$, and writing $c(g)$ for $c(\tilde{g})$ we have $c(g)=2+\zeta+\zeta^{-1}$ where $\zeta \in \mathbf{C}$ is a primitive nth root of unity.

(b) Assume that $g$ has finite order. Then

$g$ has order $1,2,3,4,5 \Longleftrightarrow c(g)=4,0,1,2, \frac{3 \pm \sqrt{5}}{2}$, respectively.

Proof (a) Any two lifts of $g$ to $\mathrm{GL}_{2}(\mathbf{C})$ differ by a non-zero scalar, from which it is easy to check that $c(\tilde{g})$ depends only on $g$. If $\tilde{g}$ is any lift of $g$ to $\mathrm{GL}_{2}(\mathbf{C})$ then $\tilde{g}$ must be diagonalisable (or else $g$ would have infinite order) and if the eigenvalues are $\lambda$ and $\mu$ then the order of $g$ in $\mathrm{PGL}_{2}(\mathbf{C})$ equals the multiplicative order of $\lambda / \mu$ in $\mathbf{C}^{\times}$. Because $g$ has order $n$, we must have $\lambda=\mu \zeta$ for $\zeta$ a primitive $n$th root of unity, and now everything follows from a direct calculation.

(b) This follows easily from $(a)$.

Now let $f$ be a newform of weight one, level $N$ and character $\chi$. By Lemma 1 (a) we see that for a prime $p \nmid N$ the order of the element $\bar{\rho}_{f}\left(\operatorname{Frob}_{p}\right)$ is uniquely (and explicitly) determined by

$$
c\left(\rho_{f}\left(\operatorname{Frob}_{p}\right)\right)=\frac{a_{p}^{2}}{\chi(p)} .
$$

Moreover, when $f$ is exotic the values of $c\left(\rho_{f}\left(\right.\right.$ Frob $\left.\left._{p}\right)\right)$ which can occur, and corresponding orders of $\bar{\rho}_{f}\left(\right.$ Frob $\left._{p}\right)$, are given in Lemma 1 (b). We shall use this to separate out the exotic forms into those with $A_{4}, S_{4}$ and $A_{5}$ projective images.

Assume now that $f$ is an exotic newform, and let $S$ be a set of primes $p$ each not dividing $N$ (for our tests we take $S$ to be the set of all such primes up to some bound). Then we see immediately from our lemma that:

$$
\begin{aligned}
& f \text { is an } A_{4} \text { form } \Longrightarrow\left\{a_{p}^{2} / \chi(p) \mid p \in S\right\} \subseteq\{0,1,4\} . \\
& f \text { is an } S_{4} \text { form } \Longrightarrow\left\{a_{p}^{2} / \chi(p) \mid p \in S\right\} \subseteq\{0,1,2,4\} . \\
& f \text { is an } A_{5} \text { form } \Longrightarrow\left\{a_{p}^{2} / \chi(p) \mid p \in S\right\} \subseteq\left\{0,1, \frac{3 \pm \sqrt{5}}{2}, 4\right\} .
\end{aligned}
$$

For sufficiently large $S$ these inclusions will all become equalities, and these three implications can then be used to determine the projective image of an exotic form. The problem with this is that quantifying what we mean by "sufficiently large" requires an effective version of the Cebotarov density theorem and is likely to lead to an impractical test.

In practice $S_{4}$ and $A_{5}$ forms will soon reveal themselves, by the existence of a small prime $p \in S$ such that $a_{p}^{2} / \chi(p)=2$ or $\frac{3 \pm \sqrt{5}}{2}$, respectively. The trickier question is:

Given a suspected $A_{4}$ form, that is an exotic one with $\left\{a_{p}^{2} / \chi(p) \mid p \in S\right\}=\{0,1,4\}$, how do we rigorously rule out the possibility that $f$ is an $S_{4}$ form or $A_{5}$ form?

We first explain an efficient algorithm which can be used to show that a suspected $A_{4}$ form $f$ is not an $S_{4}$ form.

Proposition 2 Suppose $f$ is an $S_{4}$ form and $\rho_{f}$ cuts out the extension $\operatorname{Gal}\left(M_{f} / \mathbf{Q}\right)$. Then there exists a quadratic extension $K / \mathbf{Q}$ contained within $M_{f}$ such that for every element $\sigma$ of $\operatorname{Gal}\left(M_{f} / \mathbf{Q}\right)$ which is not in $\operatorname{Gal}\left(M_{f} / K\right)$, the order of $\bar{\rho}_{f}(\sigma)$ is either 2 or 4. 
Proof This follows immediately by Galois theory from the fact that every element of $S_{4}$ which is not in the index 2 subgroup $A_{4}$ has order 2 or 4.

Thus if $f$ is an $S_{4}$ form, then by Lemma 1 (b) and Proposition 2 there is a quadratic field $K$ of discriminant dividing $N$ such that for every prime $p \nmid N$ which is inert in $K$ we have $a_{p}^{2} / \chi(p) \in\{0,2\}$. So given a suspected $A_{4}$ form, we can rule out that it is an $S_{4}$ form by, for each of the finitely many quadratic fields of discriminant dividing $N$, finding a prime $p$ not dividing the level of $f$ which is inert in $K$ and for which $a_{p}^{2} / \chi(p) \neq 0$. This is in practice very easy to do.

Recall that the coefficient field of $f$ (that generated by its Fourier coefficients) is the extension of $\mathbf{Q}(\chi)$ generated by the coefficients $a_{p}$ for $p$ less than the "Sturm bound" $\frac{1}{12}\left[\mathrm{SL}_{2}(\mathbf{Z}): \Gamma_{0}(N)\right]$ in level $N$. We use the coefficient field to give a practical way of proving that a suspected $A_{4}$ form $f$ is not an $A_{5}$ form.

Proposition 3 Suppose $f$ is an $A_{4}$ form and $f$ has character of order coprime to 5. Then the coefficient field of $f$ does not contain $\sqrt{5}$.

Proof Let $G$ be the image of $\bar{\rho}_{f}$, so $G$ is a subgroup of $\mathrm{PGL}_{2}(\mathbf{C})$ isomorphic to $A_{4}$. The pre-image of $G$ in the degree 2 cover $\mathrm{SL}_{2}(\mathbf{C})$ of $\mathrm{PGL}_{2}(\mathbf{C})$ is then a group $\tilde{G}$ of order 24 , and if $Z$ denotes the scalar matrices in $\mathrm{GL}_{2}(\mathbf{C})$ then the image of $\rho_{f}$ must be contained within the group $Z \tilde{G}$. Furthermore, because the determinant of $\rho_{f}$ equals the character of $f$ and in particular has order $d$ prime to 5, the image of $\rho_{f}$ must be contained within $\mu_{2 d} \tilde{G}$ where $\mu_{2 d}$ denotes the $2 d$ th roots of unity within $Z$. In particular the image of $\rho_{f}$ must have order prime to 5. If $f=\sum a_{n} q^{n}$ then this means that each $a_{p}$ is a sum of at most 2 roots of unity of order prime to 5 (this is true even at the bad primes: by local-global compatibility the $a_{p}$ in this case equals the trace of Frobenius on the inertial invariants) and the field generated by the $a_{p}$ and the values of $\chi$ is hence unramified at 5 .

Now if $f$ is an $A_{5}$ form then the coefficient field of $f$ must contain $\sqrt{5}$, because by the Cebotarev density theorem there will be primes $p$ with $a_{p}=\frac{3 \pm \sqrt{5}}{2}$. So given a suspected $A_{4}$ form $f$ with character order coprime to 5 we can ruled out that it is an $A_{5}$ form by checking the coefficient field does not contain $\sqrt{5}$. This was enough for our purposes, since all suspected $A_{4}$ forms of level $N \leq 1500$ had character of order coprime to 5 .

However there are $A_{4}$ forms whose field of coefficients contains $\sqrt{5}$. (For example take an $A_{4}$ form and twist it by a Dirichlet character to ensure that it has character of order a multiple of 5. Then the coefficient field of the twisted form will contain $\mathbf{Q}\left(\zeta_{5}\right)$ and this contains $\sqrt{5}$.) We could fix this problem however, were it ever to occur, using the following observation. If $f$ is any eigenform then some twist $f^{\prime}=f \otimes \xi$ of $f$ will have character $\chi^{\prime}$ of order coprime to 5 (if $f$ has character $\chi$ of order $5^{d} e$ with $d \geq 1$ and $5 \nmid e$ then twist $f$ by $\chi^{\left(5^{d}-1\right) / 2}$ for example). The point of this twisting is that if $f$ really is an $A_{4}$ form then so is $f^{\prime}$, and applying Proposition 3 to $f^{\prime}$ we see that the coefficient field of $f^{\prime}$ will be unramified at 5 .

Remark 4 This test would need rather many Fourier coefficients however, up to the Sturm bound in level $N$ cond $(\xi)^{2}$ where cond $(\xi)$ is the conductor of $\xi$. We had computed the Fourier expansions modulo $O\left(q^{10,000}\right)$ but even so felt fortunate this twisting trick was not required.

Open Access This article is distributed under the terms of the Creative Commons Attribution 4.0 International License (http://creativecommons.org/licenses/by/4.0/), which permits unrestricted use, distribution, and reproduction in any medium, provided you give appropriate credit to the original author(s) and the source, provide a link to the Creative Commons license, and indicate if changes were made. 


\section{Appendix A: Accompanying data and code}

The webpage [2] should be considered an electronic appendix to this paper.

\section{References}

1. Bosma, W., Cannon, J., Playoust, C.: The Magma algebra system. I. The user language. J. Symbolic Comput. 24(3-4), 235-265 (1997). [Computational algebra and number theory (London, 1993)]

2. Buzzard, K., Lauder, A.: A computation of modular forms of weight one and small level (webpage). http://people.maths.ox.ac.uk/lauder/weight1 (2016)

3. Buhler, J.P.: Icosahedral Galois Representations. Lecture Notes in Mathematics, vol. 654. Springer, Berlin (1978)

4. Buzzard, K.: Computing weight one modular forms over $\mathbb{C}$ and $\overline{\mathbb{F}} p$. In: Computations with Modular Forms. Contrib. Math. Comput. Sci., vol. 6, pp. 129-146. Springer, Cham (2014)

5. The Sage Developers.: Sagemath, The Sage Mathematics Software System (Version 6.4.1). http://www. sagemath.org (2014)

6. Darmon, H., Lauder, A., Rotger, V.: Stark points and $p$-adic iterated integrals attached to modular forms of weight one. Forum Math. Pi, 96 (2015)

7. Darmon, H., Lauder, A., Rotger, V.: Gross-stark units and $p$-adic iterated integrals attached to modular forms of weight one. Ann. Sci. Math. Què. 40(2), 325-354 (2016)

8. Deligne, P., Serre, J.-P.: Formes modulaires de poids 1. Ann. Sci. École Norm. Sup. (4) 7(1974), 507-530 (1975)

9. Frey, G. (ed.): On Artin's conjecture for odd 2-dimensional representations. In: Lecture Notes in Mathematics, vol. 1585. Springer, Berlin (1994)

10. Khare, C., Wintenberger, J.-P.: Serre's modularity conjecture. I. Invent. Math. 178(3), 485-504 (2009)

11. The LMFDB Collaboration.: The L-functions and modular forms database. http://www.lmfdb.org (2013)

12. Schaeffer, G.: The hecke stability method and ethereal forms. Ph.D. thesis, University of California, Berkeley (2012)

13. Stark, H.M.: Class fields and modular forms of weight one. Modular functions of one variable, V (Proc. Second Internat. Conf., Univ. Bonn, Bonn, 1976), pp. 277-287. Springer, Berlin (1977). (Lecture Notes in Math., Vol. 601) 